\title{
Changes in levothyroxine pharmacokinetics following bariatric surgery in obese hypothyroid patients
}

\author{
Feyzi Gokosmanoglu' ${ }^{1}$ Attila Onmez ${ }^{2}$ \\ ${ }^{1}$ Department of Endocrinology, Medical Park Hospital, Ordu, Turkey \\ ${ }^{2}$ Department of Internal Medicine, Medical Faculty, Duzce University, Duzce, Turkey
}

\begin{abstract}
Aim: Levothyroxine (L-T4) requirements in obese hypothyroid patients receiving L-T4 therapy decrease following bariatric surgery. L-T4 absorption is also thought to decrease after surgery. The purpose of this study was to evaluate L-T4 requirements in hypothyroidism cases before and after weight loss through bariatric surgery.

Methods: Seventy-six cases of hypothyroidism receiving L-T4 replacement therapy and with a body mass index over $40 \mathrm{~kg} / \mathrm{m} 2$ were included in the study. Patients losing at least $10 \%$ of basal body weight following bariatric surgery were assessed in terms of thyroid hormone levels and L-T4 requirements over follow-up of at least one year. The L-T4 requirements of patients in whom euthyroidism was achieved were compared in terms of bariatric surgery procedures and hypothyroidism etiology.

Results: Seventy-six patients (56 women, 20 men) with a mean age of 38 years (18-51) were included in the study. Mean weight before bariatric surgery was $121.6 \pm 6.8 \mathrm{~kg}$, and mean body mass index was $49.5 \pm 1.6$ $\mathrm{kg} / \mathrm{m}^{2}$. Euthyroidism was confirmed at pre- and post-bariatric surgery evaluation. No statistically significant postoperative changes were determined in thyroid-stimulating hormone or free thyroxine-3 and $-4(p>0.05)$. A statistically insignificant decrease was observed in L-T4 dosages after surgery in cases of Hashimoto's thyroiditis $(p=0.064)$. A statistically significant decrease was determined in L-T4 dosages in cases of nonHashimoto hypothyroidism $(p=0.001)$. L-T4 requirements decreased in both surgical procedures $(p=0.001)$ Conclusion: Postoperative L-T4 requirements decrease with weight loss. In addition, no decrease appeared to occur in L-T4 absorption following the surgical procedures in this study.
\end{abstract}

Keywords: Obesity, hypothyroidism, bariatric surgery, levothyroxine.

Dr. Attila Onmez,

Department of Internal Medicine, Duzce University, Medical Faculty, Duzce, Turkey

E-mail: attilaonmez@gmail.com

Received: 2020-08-26 / Revised: 2020-09-06

Accepted: 2020-10-13 / Published online: 2021-01-01

\section{Introduction}

Obesity is one of the most important presentday health risks. Hypothyroidism is commonly observed in obese populations [1]. Clinical and subclinical hypothyroidism leads to weight gain due to a decrease in basal metabolism rates. According to common perception, hypothyroidism is responsible for obesity. However, establishing a causal link between the two is controversial. Recent observations indicate that changes in thyroidstimulating hormone (TSH) may well be secondary to obesity. At the same time, the replacement dose of Levothyroxine (L-T4) can be modified [2]. 
Drug absorption may be deficient in association with the type of bariatric surgery performed. Drug absorption deficiency can lead to an increased L-T4 requirement in hypothyroid patients. However, the data concerning this are inconsistent [3]. Research has shown that obese hypothyroid patients require higher L-T4 dosages than normal weight individuals [4]. LT4 dosages decrease following weight loss [5]. However, it may be necessary to increase the dosages due to the possibility of resectionrelated reduced drug absorption.

Decreased L-T4 requirements have recently been shown in the majority of hypothyroid patients in line with weight loss achieved with modern bariatric surgery. The purpose of the present study was to investigate the effect of weight loss following bariatric surgery performed using mini-gastric bypass and sleeve gastrectomy procedures on L-T4 requirements in hypothyroid patients.

\section{Materials and Methods}

This study was performed in compliance with the Declaration of Helsinki and was approved by the Ethics Committee of the Medicana International Samsun Hospital, Turkey (12.08.2020-9/7109).

The research concerned 76 patients. The study group occurred of adult (over 18 years) hypothyroid patients (Hashimoto's thyroiditis, thyroidectomy, radioactive iodine ablation therapy, congenital hypothyroidism, central hypothyroidism, post-subacute thyroiditis, or infiltrative causes) receiving L-T4 replacement therapy under follow-up in our endocrinology and obesity surgery department, with body mass index exceeding $40 \mathrm{~kg} / \mathrm{m} 2$ (class 3 obesity), and undergoing bariatric surgery. Patients were assessed using thyroid gland ultrasonography in order to avoid diagnostic confusion.
Patients with known thyrotoxicosis during screening, with primary endocrine/systemic disease, any inflammatory disease other than Hashimoto's thyroiditis, celiac disease and malabsorption, inflammatory gastrointestinal diseases, cancer, history of use of other medications reducing drug absorption, or receiving radiotherapy to the neck and head region were excluded. In the light of the study aims, patients achieving weight loss of at least $10 \%$ from baseline following bariatric surgery were evaluated in terms of thyroid hormone levels and LT-4 requirements initially and over a 12-month follow-up period at intervals of 2-3 months.

Patients were given detailed instruction concerning L-T4 use and asked to take the drug as described one hour before breakfast. The importance of the timing of L-T4 use was emphasized, particularly in cases using multivitamins and proton pump inhibitors in the postoperative period. Cases using the drug irregularly or frequently forgetting to take L-T4 were placed under close monitoring, and thyroid hormones were evaluated during periods when L-T4 was used regularly. If TSH values were below $2.5 \mathrm{mIU} / \mathrm{L}$ in cases losing more than $10 \%$ of body weight following bariatric surgery, the L-T4 dosage was reduced by $25 \%$. The dosage was decreased by $30 \%$ in cases with TSH below $1 \mathrm{mIU} / \mathrm{L}$ at postoperative follow-up.

Hormonal data for TSH (normal range 0.35$4.94 \mathrm{mIU} / \mathrm{L}$ ), free thyroxine-4 (fT4; normal range: $\quad 9.01-19.05 \mathrm{pmol} / \mathrm{L}), \quad$ free triiodothyronine (fT3; normal range: 3.5-8.0 $\mathrm{pmol} / \mathrm{L}$ ), albumin (normal range: 3.5-5.5 g/dL), thyroglobulin antibody (TgAb; normal range: 0-4.11 $\mathrm{lU} / \mathrm{mL})$, and thyroid peroxidase antibody (TPOAb; normal range: 0-5.61 $\mathrm{IU} / \mathrm{mL}$ ) levels were investigated using automated immune chemiluminometric assay 
(ICMA) kits (Monmouth, UK). Thyroid ultrasonography was applied using a high resolution device (Philips Medical system, USA) equipped with a 5-12 $\mathrm{MHz}$ broadband linear array probe. All procedures were performed by an experienced operative.

\section{Statistical analysis}

Data analysis was performed on SPSS version 21.0 software (SPSS Inc., Chicago, USA). Quantitative parametric data were expressed as mean plus standard deviation (SD), and quantitative non-parametric data as median values with minimum and maximum. The Kolmogorov Smirnov and Shapiro-Wilk test was used to analyze the distribution of variables. For non-parametric data, comparisons between different groups were performed using the Mann-Whitney $U$ test, while the independent- $t$ test was used to compare parametric data between the groups.

\section{Results}

Seventy-six patients (56 women, 20 men) with a median age of 38 years (18-51) were included in the research. Mini-gastric bypass was performed in 52 cases, and sleeve gastrectomy in 24. Postoperative follow-up time was 2.4 (1.7-3.6) years. Hypothyroidism associated with the surgical procedure was present in 28 cases, Hashimoto's thyroiditis in 32 , and hypothyroidism associated with other causes (radioactive iodine [RAI] intake, congenital hypothyroidism, post-subacute thyroiditis, or infiltrative causes) in 16. Patients' mean weight before bariatric surgery was $121.6 \pm 6.8 \mathrm{~kg}$, and mean body mass index was $49.5 \pm 1.6 \mathrm{~kg} / \mathrm{m}^{2}$. Mean weight after bariatric surgery decreased to $94.6 \pm 5.6 \mathrm{~kg}$ and mean body mass index to $33.3 \pm 1.4 \mathrm{~kg} / \mathrm{m}^{2}$. Cases' pre- and postoperative characteristics are shown in Table 1.

All bariatric surgery procedures were performed using standard techniques by an experienced bariatric surgeon. Euthyroidism was confirmed at pre- and post-bariatric surgery checks. No statistical postoperative changes were determined in levels of the thyroid hormones TSH, fT4 or fT3 ( $p>0.05)$. We found a statistically significant decrease in L-T4 dosages in parallel with weight loss after bariatric surgery in cases diagnosed with hypothyroidism developing after bariatric surgery and other hypothyroidism cases (RAI intake, congenital hypothyroidism, postsubacute thyroiditis, and infiltrative causes) $(p=0.001)$. We also determined a nonsignificant decrease in L-T4 dosages in cases of Hashimoto's thyroiditis $(p=0.064)$. The decrease in L-T4 dosages was highly significant in both surgical procedures $(p=0.001)$. The findings are shown in Table 2 . Albumin levels also decreased, particularly in the mini-gastric bypass cases ( $p=0.047$ ).

Table 1. Preoperative characteristics of the patients.

\begin{tabular}{|l|c|}
\hline Parameters & $\mathbf{n}=\mathbf{7 6}$ \\
\hline Age (years), median(min-max) & $38(18-51)$ \\
\hline Sex & $56(73)$ \\
Female, $\mathrm{n}(\%)$ & $20(27)$ \\
Male, $\mathrm{n}(\%)$ & $121.6 \pm 6.8$ \\
\hline Weight (kg), mean $\pm \mathrm{SD}$ & $49.5 \pm 1.6$ \\
\hline BMI, kg/m ${ }^{2}$ & \\
\hline Etiology of hypothyroidism & 28 \\
Post-thyroidectomy & 32 \\
Hashimoto's thyroiditis & 16 \\
Other ${ }^{1}$ & \\
Type of bariatric surgery & 52 \\
Mini-gastric bypass & 24 \\
\hline Sleeve gastrectomy & \\
\hline
\end{tabular}

BMI: Body Mass Index, SD: Standard Deviation

${ }^{I} R A I$ intake, congenital hypothyroidism, postsubacute thyroiditis, infiltrative causes. 
Table 2. Changes in thyroid function and L-thyroxine dosages following bariatric surgery.

\begin{tabular}{|c|c|c|c|}
\hline Parameters & $\begin{array}{c}\text { Before bariatric } \\
\text { surgery }\end{array}$ & $\begin{array}{l}\text { After bariatric } \\
\text { surgery }\end{array}$ & $p$ \\
\hline Weight $(\mathrm{kg})($ mean $\pm \mathrm{SD})$ & $121.6 \pm 6.8$ & $94.6 \pm 5.6$ & 0.001 \\
\hline BMI $\left(\mathrm{kg} / \mathrm{m}^{2}\right)$ & $49.5 \pm 1.6$ & $33.3 \pm 1.4$ & 0.001 \\
\hline TSH (mIU/L) & $2.1 \pm 0.5$ & $1.9 \pm 0.4$ & 0.652 \\
\hline fT4 (pmol/L) & $13.1 \pm 0.8$ & $14.9 \pm 0.6$ & 0.860 \\
\hline fT3 (pmol/L) & $4.6 \pm 0.4$ & $4.5 \pm 0.8$ & 0.972 \\
\hline $\begin{array}{c}\text { L-thyroxine, mcg/day, dose } \\
\text { Surgical hypothyroidism } \\
\text { Hashimoto's thyroiditis } \\
\text { Other hypothyroidism }{ }^{1}\end{array}$ & $\begin{array}{l}149.3 \pm 46.7 \\
152.5 \pm 52.3 \\
140.5 \pm 42.5\end{array}$ & $\begin{array}{l}112.5 \pm 32.4 \\
145.9 \pm 36.8 \\
115.6 \pm 31.9\end{array}$ & $\begin{array}{l}0.001 \\
0.064 \\
0.001\end{array}$ \\
\hline Albumin, $\mathrm{g} / \mathrm{dL}$ & $4.7 \pm 0.6$ & $4.2 \pm 0.8$ & 0.043 \\
\hline $\begin{array}{l}\text { Type of bariatric surgery, } \\
\text { L-thyroxine, mcg/day, dose } \\
\text { Mini-gastric bypass } \\
\text { Sleeve gastrectomy }\end{array}$ & $\begin{array}{l}148.4 \pm 49.2 \\
150.6 \pm 51.5\end{array}$ & $\begin{array}{l}125.2 \pm 35.7 \\
118.9 \pm 38.3\end{array}$ & $\begin{array}{l}0.001 \\
0.001\end{array}$ \\
\hline
\end{tabular}

TSH, thyroid-stimulating hormone; fT4, free-T4; fT3, free-T3; RAI, radioactive iodine; SD: Standard Deviation. ${ }^{I} R A I$ intake, congenital hypothyroidism, post-subacute thyroiditis, infiltrative causes.

\section{Discussion}

L-T4 dosage requirements decrease in parallel with weight loss in obese cases diagnosed with hypothyroidism. Loss of body mass eliminates anxiety concerning a decrease in L-T4 absorption resulting from the operation. Newly evidence [5] and our own study data show that thyroid hormone requirements may decrease with weight loss in obesity.

A few studies have determined significant decreases in TSH and fT3 levels with weight loss after obesity surgery. Weight loss also reduces thyroid hormone requirements [5]. Similarly in the present study, we found a statistically significant decrease in L-T4 requirements in association with weight loss. L-
T4 dosage requirements decrease in parallel with weight loss to yield similar TSH levels in both types of surgery. In our previous study we observed that TSH levels decreased in correlation with insulin resistance following bariatric surgery [6]. Juiz-Valiña et al. [7] also reported similar findings. We therefore think that L-T4 requirements decreased through a similar pathway to the decrease in insulin resistance occurring with weight loss in association with thyroid hormone resistance in obese patients. It possibly concluded that weight loss restores thyroid hormone homeostasis. Research has shown that severely obese individuals may require higher L-T4suppressing or replacement therapy than 
normal weight subjects in association with impairment of the L-T4 pharmacokinetic parameters [8].

Decreased thyroid gene expression (particularly TSH-receptors) has been reported in visceral adipose tissue and subcutaneous in obese patients [9]. The decrease in adipose tissue in parallel with weight loss following obesity surgery results in corresponding changes in serum TSH and thyroid hormone levels. These changes show that adipocytes are involved in the regulation of thyroid hormones. Our research revealed that L-T4 dose requirements decreased in parallel with weight loss in cases of surgical hypothyroidism and other hypothyroidism cases. This finding suggested that the level of adverse effects on drug absorption caused the decrease in gastric acid in the sleeve gastrectomy group can be overlooked. In the mini gastric bypass cases, however, approximately $1 / 3$ of the length of the intestine was bypassed. This method may have permitted sufficient drug absorption through the non-bypass intestine.

Research has shown that L-T4 requirements decrease in the postoperative period. The principal correlation shown in research is between weight loss and dosage [1]. The principal L-T4 fluid formulation has been shown to be capable of preventing the problem of malabsorption after bariatric surgery in malabsorptive operations (Roux-en-Y gastric bypasses and biliary pancreatic diversions) [10]. Our findings showed no L-T4 malabsorption in sleeve gastrectomy and mini gastric bypasses. Loss of both adipose and nonadipose mass following these operations may result in a decrease in postoperative L-T4 requirements by preventing absorptive effects resulting from surgery [11]. These studies have shown no decrease in L-T4 absorption in Rouxen-Y bypasses. Small but significantly delayed
L-T4 absorption has been observed after surgery in these cases [12].

Research involving sleeve gastrectomy and mini gastric bypasses has shown no significant L-T4 absorption in the gut, duodenum, or upper part of the jejunum [13]. These are bypassed or removed in bariatric surgery. These studies offer powerful support for our own findings. LT4 dosage requirements also decrease in parallel with weight loss. In contrast, although we observed a decrease in drug requirements in both types of operation in hypothyroidism cases associated with Hashimoto's thyroiditis, this was not statistically significant. This variation may be due to an increase dose requirement deriving from the chronic progressive course of Hashimoto's thyroiditis. Alternatively, these operations may have resulted in additional absorption insufficiency due to Hashimoto's thyroiditis being frequently accompanied by celiac disease, inflammatory bowel disease, and autoimmune gastric diseases. We think that these factors may account for the decreased LT4 dosage requirements determined in other cases not being observed in Hashimoto's thyroiditis cases.

\section{Conclusion}

Both adipose and non-adipose body mass decrease with significant weight loss achieved with bariatric surgery. Marked weight loss results in a decrease in postoperative L-T4 requirements. This decrease is more powerful than the effect of resection on absorption. This study shows that sleeve gastrectomy and mini gastric bypasses do not result in L-T4 malabsorption. This study adds new findings to the actual evidence regarding changes caused by weight loss in thyroid morphology.

Funding: There is no financial support and sponsorship 
Conflict of Interest: The authors declare that they have no conflict of interest.

Ethical statement: This study was performed in compliance with the Declaration of Helsinki and was approved by the Ethics Committee of the Medicana International Samsun Hospital, Turkey (Date and decision number: 12.08.2020-9/7109).

\section{ORCID iD of the author(s)}

Feyzi Gokosmanoglu / 0000-0002-6432-8668

Attila Onmez / 0000-0002-7188-7388

Copyrights: (C)2021@author (s).

This is an open access article distributed under the terms of the Creative Commons Attribution License (CC BY 4.0), which permits unrestricted use, distribution, and reproduction in any medium, provided the original author(s) and source are credited and that the original publication in this journal is cited, in accordance with accepted academic practice. No use, distribution or reproduction is permitted which does not comply with these terms.

\section{References}

[1]Bernadette B. Thyroid and Obesity: An Intriguing Relationship. J Clin Endoc Metabol. 2010;8(1):3614-17.

[2]Sanyal D, Raychaudhuri M. Hypothyroidism and obesity: An intriguing link. Indian J Endocrinol Metab. 2016;20(4):554-57.

[3]Gadiraju S, Lee CJ, Cooper DS. L-T4 Dosing Following Bariatric Surgery. Obes Surg. 2016;26(10):2538-42.

[4]Longhi S, Radetti G. Thyroid function and obesity. J Clin Res Pediatr Endocrinol. 2013;5(1):40-44.

[5]Zendel A, Abu-Ghanem Y, Dux J, et al. The Impact of

Bariatric
Surgery on Thyroid Function and Medication Use in Patients with Hypothyroidism. Obes Surg. 2017;27(8):2000-4.

[6]Gokosmanoglu F, Aksoy E, Onmez A, et al. Thyroid Homeostasis After Bariatric Surgery in Obese Cases. Obes Surg. 2020;30(1):274-78.

[7]Juiz-Valiña P, Cordido M, Outeiriño-Blanco $\mathrm{E}$, et al. Central resistance to thyroid hormones in morbidly obese subjects is reversed after bariatric surgery-induced weight loss. J Clin Med. 2020;9(2):359.

[8]Guan B, Chen Y, Yang J, et al. Effect of bariatric surgery on thyroid function in obese patients: a systematic review and meta-analysis. Obes Surg. 2017; 27(12):3292-305.

[9]Yang J, Gao Z, Yang W, et al. Effect of sleeve gastrectomy on thyroid function in chinese euthyroid obese patients. Surg Laparose Endosc Percutan Tech. 2017;27(4):66-68.

[10] Michalaki MA, Gkotsina MI, Mamali I, et al. Impaired pharmacokinetics of L-T4 in severely obese volunteers. Thyroid. 2011;21(5):477-81.

[11] Nannipieri M, Cecchetti F, Anselmino M, et al. Expression of thyrotropin and thyroid hormone receptors in adipose tissue of patients with morbid obesity and/or type 2 diabetes: effects of weight loss. Int $\mathbf{J}$ Obesity. 2009;33(9):1001-6.

[12] Fallahi P, Ferrari SM, Camastra S, et al. TSH normalization in bariatric surgery patients after the switch from L-T4 in tablet to an oral liquid formulation. Obes Surg. 2017;27(1):78-82.

[13] Santini F, Pinchera A, Marsili A, et al. Lean body mass is a major determinant of L-T4 dosage in the treatment of thyroid diseases. $\mathrm{J}$ Clin Endocrinol Metab. 2005; 90(1):124-27. 
[14] Rubio IG, Galrão AL, Santo MA, et al. L-T4 absorption in morbidly obese patients before and after Roux-En-Y gastric bypass (RYGB) surgery. Obes Surg. 2012; 22(2):253-58.

[15] Gkotsina M, Michalaki M, Mamali I, et al. Improved L-T4 pharmacokinetics after bariatric surgery. Thyroid. 2013;23(4):414-19. 